\section{Cells evolving}

\author{
T. Cavalier-Smith
}

Blueprint for a Cell: The Nature and Origin of Life. By C. de Duve. Patterson/Portland: 1991. Pp.275. $\$ 19.95$, £17.95.

For almost 3,000 million years living organisms were essentially unicellular: all evolution was cell evolution. But, curious though it may seem, much less has been written about the evolution of cells than about the relatively brief chemical evolution that preceded it or the recent evolution of multicellular organisms. De Duve's attractive and well-written book, Blueprint for a Cell, is therefore most welcome. The author is best known for 'discovering', or rather establishing the true nature of, lysosomes and peroxisomes. His earlier A Guided Tour of the Living Cell (Scientific American Books, 1984) was one of the best semipopular introductions to cell biology. The present book also deserves to be widely read.

Its first part outlines the most essential features of living organisms and how these are differently expressed in bacterial and eukaryotic cells. This leads to a reconstruction of the ancestral cell, in preparation for the second part which attempts to suggest how this ancestral cell may have originated. Part one is a rather well-balanced treatment that generally avoids excessive bias in one or other fashionable directions. But it does somewhat overemphasize the living state, at the expense of the processes of cell reproduction, which are absolutely central to an understanding of the dynamics of cell evolution.

The chapter on bacteria does not even mention the vital role of the rigid cell wall in cell growth and division and in providing a rigid framework for the segregation of DNA. Therefore the drastic implications of its loss for the origin of the eukaryotic cell are only partially appreciated. When discussing the origin of mitosis de Duve suffers from a widespread misconception, often propagated in even the best textbooks, that dinoflagellate chromosomes are not attached to spindle microtubules, and that segregation by membrane growth was a primitive eukaryote condition. In fact there is no reason to think that fluid lipid bilayers are able to segregate DNA in any organism. Some sort of skeleton, whether an exoskeleton as the bacterial wall or an endoskeleton as the eukaryote cytoskeleton, are essential for the reliable transmission of genetic information from generation to generation. The changeover from the bacterial to the eukaryotic pattern must have been far more traumatic than de Duve recognizes.

The whole book suffers from a gradualistic and a deterministic bias. The author does not recognize the evolutionary importance of historical contingency or of long periods of stasis followed by sudden change. Far too little note is taken of the fossil record, which suggests that eukaryotes evolved only about half as long ago as bacteria $\left(1.7 \times 10^{9}\right.$ years ago, not $3.5-2.3 \times 10^{9}$ years ago as the author implies), and in no way supports the author's view that the origin of eukaryotes and of ourselves was inevitable. To dismiss chance and historical accidents as miracles and to allow only strict determinism is philosophically unsound.

Although the author has been overinfluenced by uncalibrated gradualistic 'molecular-clock' ideas in his dating, he rightly realizes that the most recent common ancestor of all life cannot possibly have been a 'nebulous' progenote, but must have been a highly developed bacterial cell. He criticizes my view that this was eubacterial-not archaebacterial-in its lipids because he thinks that acylester lipids could have been invented twice; yet he inconsistently invokes possible biophysical reasons to explain why in archaebacteria a changeover from isoprenoidalether to acylester lipids never occurred. If it never occurred for such reasons in archaebacteria, why postulate it twice independently in eukaryotes and eubacteria? I am tempted to conclude that de Duve's reluctance to accept that the ancestral cell was a eubacterium is because he prefers to suppose it was an acid-resistant, thermophilic sulphur-dependent archaebacterium, because this appears to bolster his dubious theory for the prebiotic origin of a bioenergetic system based on thioesters.

In contrast to the first part of the book which is basically sound, part two is wildly speculative and fundamentally flawed in its two central theses. The most fundamental of these is the idea that chemical evolution produced a complete protometabolism of metabolic pathways catalysed by noninformed oligopeptides before the origin of replication and translation; moreover this protometabolism, which is incredibly held to have included essentially all the currently existing biosynthetic pathways and therefore all the raw materials for life, was preserved in some way during the changeover from uninformed to coded protein synthesis. Neither half of this central thesis is remotely plausible. Yet in one sense this general line of thinking ought to be pursued, and studied experimentally, because it must surely be the case that replication and translation originated, not in almost pure systems of the sort currently studied in the laboratory but in incredibly complex mixtures of molecules produced by the factors (mass action and crude prebiotic catalysts) that de Duve so lucidly describes. Even if, contrary to de Duve's hopeful assertions, this 'protometabolism' did not in fact give rise directly to modern metabolism it might well, as he argues, have provided an essential prerequisite for the origin of the constituents of the 'RNA world' or some yet more primitive self-replicating system.

De Duve's second thesis is that his protometabolism evolved a complete bioenergetic system that was initially based on thioesters, and later evolved substrate-level phosphorylation, all before the origin of replication, translation or the first cell. He rejects the more conventional idea that bioenergetics started with pyrophosphate, because of the low concentration of phosphate at neutral $\mathrm{pH}$ in the presence of calcium. But because his thioester theory also would not work at neutral $\mathrm{pH}$ and low temperatures, he suggests that life began not at neutral $\mathrm{pH}$ but in hot acid (despite the instability of many key biogenic molecules under these conditions). But if one can postulate special environmental conditions for thioesters why not also for phosphates? De Duve does not explain why the low concentration of phosphate was not equally a problem for the postulated later origin of phosphorylation and nucleotides. His reasons for rejecting the widely discussed idea that the first bioenergetic system was a membrane-based photophosphorylation (not substrate-level phosphorylation) are equally unclear: perhaps it simply reflects the traditional biases of animal biochemists, which de Duve openly admits he shares.

In much of part two the selective forces that favour each postulated stage are very vague and inexplicit. For example, when discussing the origin of translation he says that it was not "the quality of the messages that counts", but of the ability to synthesize polypeptides. But though selection can directly improve replication regardless of its other phenotypic consequences this cannot be true of translation: there must be some specific benefit of the product to the system.

De Duve recognizes the importance of the association of early replicating molecules with some structure, for example a membrane, that can itself grow and divide and therefore be subject to "true darwinian selection' but is very vague as to the specific benefits of such association: if the first bioenergetic system depended on membranes, as in the phototrophic theory, the advantages are by contrast quite obvious. He favours the inside-out cell/obcell of G. Blobel and myself as a precursor for true cells, but is very vague as to the properties of his postulated protocell: in one place he assumes it was porous and in another impermeable to protons.

Even though he touches on many of the most important considerations for understanding the origin of cells there is relatively poor integration in part two between them, which is a pity because the subject of cell evolution (as opposed to the more specialized and limited molecular evolution) should above all else be integrative one. Nonetheless, though to some degree falling between two stools (too technical for the intelligent layman; insufficiently detailed or rigorously argued for the specialist), Blueprint for a Cell will be stimulating reading for a broad scientific audience.

T. Cavalier-Smith is in the Department of Botany, University of British Columbia, Vancouver, British Columbia V6T 124, Canada. 